\title{
Erratum to: U-shaped association between body mass index and proteinuria in a large Japanese general population sample
}

\author{
Yuji Sato • Shouichi Fujimoto $\cdot$ Tsuneo Konta $\cdot$ Kunitoshi Iseki · Toshiki Moriyama • \\ Kunihiro Yamagata $\cdot$ Kazuhiko Tsuruya $\cdot$ Hideaki Yoshida $\cdot$ Koichi Asahi · \\ Issei Kurahashi · Yasuo Ohashi · Tsuyoshi Watanabe
}

Published online: 28 June 2013

(C) Japanese Society of Nephrology 2013

\section{Erratum to: Clin Exp Nephrol \\ DOI 10.1007/s10157-013-0809-5}

The original version of this article unfortunately contained errors.

In the Abstract, under the heading "Methods", the number of men (median age 66 years) should be 85,183 , not 185,183 .
The online version of the original article can be found under doi:10.1007/s10157-013-0809-5.

\section{Y. Sato $(\bowtie)$}

Dialysis Division, University of Miyazaki Hospital,

Miyazaki, Japan

e-mail: ysato@fc.miyazaki-u.ac.jp

\section{S. Fujimoto}

Department of Hemovascular Medicine and Artificial Organs,

Faculty of Medicine, University of Miyazaki, Miyazaki, Japan

\section{T. Konta}

Department of Cardiology, Pulmonology, and Nephrology,

Yamagata University School of Medicine, Yamagata, Japan

K. Iseki · T. Moriyama $\cdot$ K. Yamagata $\cdot$ K. Tsuruya $\cdot$

H. Yoshida $\cdot$ K. Asahi · T. Watanabe

Steering Committee for the "Examination of the Positioning of CKD in Specific Health Check and Guidance", Tokyo, Japan

\section{Kurahashi}

Department of Planning, Information, and Management,

University of Tokyo Hospital, Tokyo, Japan

\section{Y. Ohashi}

Department of Biostatistics/Epidemiology and Preventive Health Sciences, School of Health Sciences and Nursing, University of Tokyo, Tokyo, Japan
Also in the "Methods" section, under the heading "Baseline measurement", lines 11-14 should read:

Urine dipstick results were interpreted by the medical staff at each local medical institution and recorded as (-), $( \pm),(1 \pm),(2 \pm)$, and $(3 \pm)$. 